Title: Metacognition in Functional Cognitive Disorder- a Potential Mechanism and Treatment Target.

First author: Rohan Bhome, MBBS,

Division of Psychiatry, University College London, London, UK

2nd author: Andrew McWilliams, MBBS,

1) Department of Psychological Medicine, Institute of Psychiatry, Psychology and Neuroscience, King's College London, 16 de Crespigny Park, London SE5 8AF, UK.

2) Wellcome Centre for Human Neuroimaging, University College London, UK.

3) UCL Institute of Child Health, Great Ormond Street, London, UK.

3rd author: Jonathan D Huntley, $\mathrm{PhD}$,

Division of Psychiatry, University College London, London, UK

4th author: Stephen Fleming, $\mathrm{PhD}$,

1) Wellcome Centre for Human Neuroimaging, University College London, London, UK

2) Max Planck University College London Centre for Computational Psychiatry and Ageing Research, London, UK

Last author: Robert J Howard, MD,

Division of Psychiatry, University College London, London, UK

Corresponding author: Rohan Bhome, MBBS, Division of Psychiatry, University College London, $6^{\text {th }}$ Floor Maple House, 149 Tottenham Court Road, London, W1T 7NF rohan.bhome@ucl.ac.uk, Telephone: 02031084036

Keywords: Functional Cognitive Disorder; metacognition; treatment; mechanism Word count: 3187 (with references in text) 


\begin{abstract}
Introduction: Functional Cognitive Disorder (FCD) is common. Despite this, there is no evidence-based consensus on how to treat FCD. Poor metacognitive ability has been suggested as a key mechanism underlying the disorder. We review the evidence and evaluate the proposal that strategies which improve metacognition could provide a mechanistically plausible translational therapy.
\end{abstract}

Methods: We reviewed the existing literature relating to metacognition in FCD, previous strategies to improve metacognitive ability in FCD and whether metacognitive performance can be modulated.

Results: Though limited, there is evidence to suggest that metacognition is impaired in FCD. Converging evidence from neuroimaging studies suggests that metacognitive performance can be modulated. The effectiveness of existing strategies to improve metacognition including cognitive training, psychoeducation and lifestyle interventions have been equivocal. Recently, a potential treatment option has emerged in the form of a computer-based metacognitive training paradigm.

Conclusions: There is an urgent need for effective treatments in FCD. Impaired metacognition may be a plausible therapeutic target but, in the first instance, further research is required to demonstrate deficits in "local" metacognitive ability in FCD patients when measured objectively. If so, clinical trials of interventions, such as computerised metacognitive training, are required to evaluate their effectiveness in improving FCD symptoms. 


\section{Metacognition in Functional Cognitive Disorder- a Potential Mechanism and Treatment Target.}

Functional cognitive disorder (FCD) is characterised by the experience of persistent and distressing subjective cognitive difficulties in the absence of detectable objective cognitive deficit and underlying brain pathology. Poor metacognitive ability has been suggested as a key mechanism underpinning the disorder (Bharambe and Larner, 2018a; Bhome, Huntley, Price, \& Howard, 2019; Larner, 2018b; Larner, 2018c; Metternich, Schmidtke, \& Hull, 2009; Pennington, Newson, Hayre, \& Coulthard, 2015). Metacognition refers to the ability to reflect on and monitor cognitive processes (Nelson and Narens, 1990; Fleming, Dolan and Frith, 2012) and has been quantified in the laboratory as the moment-to-moment match between subjective performance appraisal and objectively observed behaviour (Fleming and Lau, 2014). Here we review the evidence relating to metacognition in FCD and evaluate the proposal that strategies based on improving metacognition could provide a mechanistically plausible translational therapy for people with FCD.

\section{Functional cognitive disorder- a brief overview}

A growing number of patients present to health services with a primary complaint of subjective awareness of impairment of cognition, which has no objective or organic basis (Bell, Harkness, Dickson, \& Blackburn, 2015). A significant proportion of these individuals have FCD. Pennington and colleagues (Pennington, Newson et al., 2015) found that a third of patients under sixty years old seen in a dedicated memory clinic had FCD while Bharambe and Larner (Bharmabe and Larner, 2018b) identified the diagnosis in more than half of attendees in a specialist cognitive disorders clinic.

Six different but overlapping typologies of FCD have been proposed. These include cognitive symptoms in the context of affective illness, undue concern and excessive focus on normal cognitive lapses, subjective cognitive complaints that exceed what would be considered as 
normal lapses, hypochondriasis focussed on having a dementing illness, cognitive symptoms occurring co-morbidly with other functional disorders and retrograde dissociative amnesia (Stone et al., 2015). Presenting symptoms of FCD, regardless of typology, typically include complaints of "absent mindedness", concentration difficulties whilst undertaking a task, forgetting over-learnt information at a crucial moment (often only to remember it later) and prospective memory lapses (Pennington, Newson et al., 2015; Schmidtke, Pohlmann, \& Metternich, 2008; Stone et al., 2015). These symptoms often cause anxiety, anger and frustration which in turn increase the risk of further cognitive failures, propagating a reinforcing cycle that results in significant underperformance in social, work and home settings (Metternich et al., 2009). Indeed, in a recent case series more than half of all FCD patients reported that they were unemployed secondary to their illness (Bhome et al., 2019).

Characteristic clinical features of FCD which may help to distinguish it from neurological causes of cognitive impairment include memory perfectionism, excessive concern surrounding cognitive performance (Metternich et al., 2009), inconsistency between subjective cognitive difficulties and objective performance either on neuropsychological assessment or in everyday functioning, absence of underlying neuropathology and a lack of progression of symptoms (Pennington, Newson et al., 2015). Additionally, recent work has suggested that interactional styles including responding appropriately to compound questions, answering quickly and talkatively, not turning to a companion when providing details and referring back to previous answers are suggestive of FCD rather than a neurodegenerative disorder (Bailey, Poole \& Blackburn, 2018).

FCD patients commonly have co-morbid depression (Bhome et al, 2019; Pennington, Hayre, Newson, \& Coulthard, 2015; Elhadd, Bharambe, \& Larner, 2018a) and evidence of other non-cognitive functional disorders (Bharambe and Larner, 2018a; Bhome et al., 2019). Importantly, FCD should not simply be considered as the set of cognitive symptoms found in depression, sometimes referred to as 'pseudodementia', but instead be regarded as a 
discrete, though often co-morbid, disorder (Bhome et al., 2019; Pennington, Hayre et al., 2015).

Patients with FCD are likely to demonstrate subtle and non-specific cognitive deficits on neuropsychometric testing (Bhome et al., 2019; Pennington, Hayre et al., 2015) and this pattern is different from that seen in patients with neurodegenerative cognitive disorders (Wakefield et al., 2018). Teodoro and colleagues' systematically reviewed neuropsychometric profiles of patients with fibromyalgia (FM), chronic fatigue syndrome (CFS) and functional neurological disorders (FNDs) and found deficits of attention, both selective and divided, as well as slowed processing speed. They proposed that people with FCD have reduced attentional reserve resulting in slower processing speed and making them more vulnerable to distraction leading to impairment in day-to-day functioning which may not necessarily be detected on objective neuropsychometric testing ((Teodoro, Edwards, \& Isaacs, 2018).

Diagnostically, FCD falls under the categories of Conversion Disorder (also known as Functional Neurological Symptom Disorder) in DSM-V (American Psychiatric Association, 2013) and Dissociative Neurological Symptom Disorder, with cognitive symptoms (6B60.9) in the recent ICD-11 (World Health Organisation, 2018). In DSM-V there is no requirement for a prior stressor to cause the condition and diagnosis is based on internal and external inconsistencies as well as symptoms having a significant impact on functioning. Both classification systems have a separate category for dissociative amnesia which is one of the typologies described by Stone and colleagues (Stone et al., 2015). At the clinician-patient interface, however, making a diagnosis of FCD is challenging and nuanced. The extent to which clinicians investigate for an organic cause has to be cautiously balanced with the iatrogenic harm that could be caused by repeated investigations and a protracted period of diagnostic uncertainty. Two groups (Delis and Wetter, 2007; Schmidtke and Metternich, 2009) have suggested possible FCD diagnostic criteria but both have limitations which have been discussed elsewhere (Bhome et al., 2019). 
Recently, there has been a call for clinicians to make earlier and less risk-averse diagnoses of functional cognitive disorder (Stone et al., 2015), based on the presence of characteristic clinical features. Whilst this move away from FCD being a diagnosis of exclusion is timely, there is no evidence-based consensus on how to treat the increasing numbers of patients being diagnosed with FCD.

\section{Metacognitive impairment as a potential unifying mechanism in FCD}

FCD is best considered within the spectrum of FND (Pennington, Newson et al., 2015; Stone et al., 2015) in which there is a discrepancy between the individual's subjective appraisal and objective evidence of their actual performance. This has led to the hypothesis that deficits in metacognition, the ability to reflect on and monitor cognitive processes, may be a unifying mechanism underpinning FCD (Bharambe and Larner, 2018a; Bhome et al., 2019; Metternich et al., 2009; Pennington, Newson et al., 2015).

Although this hypothesis seems intuitive, there remains a question as to how a single construct can underlie such a symptomatically diverse disorder. We postulate that aetiological factors including predisposing personality traits (Metternich et al., 2009), presence of risk factors for cognitive impairment (e.g. family history of dementia, brain injury) (Bharambe and Larner, 2018a; Bhome et al., 2019), personal experience of cognitive impairment in others and the presence of psychiatric including other functional illness (Bharambe and Larner, 2018a; Bhome et al., 2019; Pennington, Hayre et al., 2015) give rise to the three central and inter-related symptoms of FCD. These are excessive concerns about cognitive performance, increased monitoring of cognitive lapses (Metternich et al., 2009), and misinterpretation of actual attentional lapses that arise due to reduced externally directed attention (Teodoro et al., 2018). We suggest that metacognitive ability normally regulates the expression of these core symptoms. For example, when metacognitive ability is impaired, there will be greater undue concern about objectively normal cognitive performance, poorer quality monitoring of cognitive lapses and increased focus upon and misinterpretation of the significance of actual attentional lapses. These related symptoms 
become intensified and lead to a reinforcing cycle, with accompanying distress and illness. Conversely, we predict that if an individual with unimpaired metacognitive ability was exposed to the same aetiological risk factors, an intact ability to reflect on and monitor cognitive processes would prevent fleeting symptoms from being reinforced and developing into an illness.

The core features, which are regulated by metacognitive ability, could be described as a "cognitive scaffold" akin to the "seizure scaffold" described by Brown and Reuber in their integrative cognitive model (ICM) of psychogenic non-epileptic seizures (Brown and Reuber, 2017). In a corresponding ICM for FCD, the activating and triggering factors are less well established because by their nature, the symptoms are often persistent rather than episodic. However, it is possible that subjective lapses secondary to misdirected attention (Teodoro et al.2018) are a negative activating event of the "cognitive scaffold". The emotional response (Metternich et al., 2010) to these lapses such as frustration, fear and anger coupled with the stress, both predating symptoms and arising as a consequence, contribute to inhibitory processing dysfunction thereby allowing the activation of the "cognitive scaffold". Central to the ICM model is the illness scaffold, in FCD we suggest that metacognitive ability underpins the "cognitive scaffold".

Existing neurobiological models of FND include three key concepts; attention towards the functional symptom, belief which refers to probabilistic representations of sensory experiences and a lack of agency for symptoms (Edwards, Fotopoulou and Parees, 2013). The Bayesian model that integrates these concepts (Edwards, Adams, Brown, Parees \& Friston, 2012) relies on a disconnect between 'top-down' predictions of a motor, sensory or cognitive process and 'bottom up' interpretation of sensory inputs. In FND, the model is dependent on an abnormal 'prior' or expectation at an intermediate hierarchical level. In FCD, the biopsychosocial aetiological factors, described above, influence the construct of this prior. Misdirected attention adds weight to this prior which then overrides any 'bottom-up' sensory input about actual cognitive functioning, giving rise to the experience of cognitive 
symptoms as predicted by the prior. In this computational model, higher order brain regions that regulate self- attention towards cognitive processes are not coupled to the predictions of the prior and so the resulting cognitive difficulties are perceived as involuntary.

The formation of an abnormal prior is absolutely paramount in the Bayesian model of FND. In FCD, the abnormal prior would be an expectation of cognitive difficulties. The prior is subject to change and we would hypothesise that it is significantly influenced by metacognition. People with impaired metacognitive ability would have far greater disparity between their expectations of cognitive ability and actual performance thereby providing further weight to them predicting poor cognitive performance in the prior.

If metacognitive abilities generalise across a range of first-order cognitive domains, this could explain why people with FCD often have subjective difficulties across a range of cognitive domains (Stone et al., 2015). A recent review (Rouault, Seow, Gillan, \& Fleming, 2018) of neuroimaging studies concluded that domain-general and domain-specific metacognitive neural circuitry are likely to co-exist. Computationally, domain-general circuitry increases efficiency by facilitating the self-appraisal of performance across tasks using a single global framework (Donoso, Collins, \& Koechlin, 2014). Additionally, performance in one domain may help to predict performance in another (Rouault et al., 2018).

Another important concept from the metacognitive literature is that of 'confidence leak' (Rahnev, Koizumi, McCurdy, D’Esposito, \& Lau, 2015), whereby confidence in appraisal of ability on one task influences confidence in another task, regardless of actual performance. Large 'confidence leaks' are maladaptive as they threaten coherent coupling between confidence and performance on tasks leading to worsening metacognitive ability (Rouault et al., 2018). In FCD, we would expect that poor confidence in one cognitive domain would quickly generalise across other cognitive domains, despite maintained objective performance, and that this, in turn, would lead to further deterioration in metacognitive performance. 


\section{Evidence for impaired metacognitive ability in FCD}

If our hypothesis is correct, impaired metacognitive ability should distinguish FCD patients from healthy individuals who share similar aetiological risk factors. Surprisingly, there has been little research directly investigating metacognitive ability in FCD. Metternich (Metternich et al., 2009) compared 39 patients with FCD diagnosed using Schmidtkes' functional memory disorder questionnaire (Schmidtke, 1995) and 38 healthy controls. There were no significant differences in objective cognitive performance (verbal memory, processing speed, premorbid intelligence) between the groups but patients with FCD had significantly $(p<0.001)$ lower memory self-efficacy (MSE) scores compared to controls (lower scores representing greater impairment in metacognition). MSE is a "global" measure of metacognition derived from three subscales of the Metamemory in Adulthood Questionnaire (MIA) (Dixon, Hultsch, \& Hertzog, 1988) which focuses on patients' perceptions of their own memory performance, change in memory and anxiety surrounding memory utilisation.

Paradise et al (Paradise, Glozier, Naismith, Davenport, \& Hickie, 2011) developed a single screening question which asks patients to appraise their memory using a five-point Likert rating scale with a response of fair or poor (2 or 1 ) being classified as positive for subjective memory complaints (SMC+). This screening tool has been used in two prospective memory clinic studies (Bharambe and Larner, 2018a; Larner, 2018c). Larner (Larner, 2018b) combined the results of these studies ( $n=130$, prevalence of non-functional cognitive impairment $46 \%$ ) and found that being SMC+ was associated with a probability of $87 \%$ for having FCD. This suggests that a poor subjective rating of objectively unimpaired cognitive performance, namely impaired metacognitive ability, is a sensitive marker for FCD.

Existing research evaluating the role of metacognitive ability in FCD has some key limitations. Firstly, while the limited evidence suggests that metacognitive deficits exist in FCD, the data are based on subjective measures of "global" metacognitive performance. Arguably, it would be "local" metacognitive sensitivity - the ability to track subtle changes in moment-to-moment cognitive performance - that is more relevant in FCD, and this construct 
can now be quantified by a combination of task-based measures and signal detection theory modelling (Fleming and Lau, 2014).

Further, existing research does not necessarily provide evidence for the direction of causality which would be required to justify our hypothesis that impaired metacognitive ability underpins FCD. Conceivably, misdirected attention could lead to subjective cognitive lapses which are then detected by hypervigilance and reinforced by predisposing factors including personality traits, affective states and personal experience of cognitive impairment in others. In this model, as the frequency and degree of subjective cognitive lapses intensify, people with FCD are likely to develop a mismatch between subject and objective measures of cognitive performance (impaired metacognitive ability).

There is an urgent need to for controlled, lab-based research investigating whether people with FCD have impaired metacognitive ability when measured objectively using recently developed techniques (Fleming and Lau, 2014). If this is confirmed, subsequent work needs to explore whether training metacognition leads to a reduction in FCD symptoms, reduced distress and improved functioning as this would support a hypothesis in which impaired metacognitive ability drives FCD symptomatology rather than being a consequence of having FCD.

\section{Potential of metacognition as a therapeutic target in FCD}

Recent converging evidence has demonstrated shared neural correlates of metacognitive performance in frontal and parietal lobes across a range of first-order tasks (Fleming, Weil, Nagy, Dolan, \& Rees, 2010; Fleming and Dolan; 2012; McCurdy et al., 2013; Baird, Smallwood, Gorgolewski, \& Margulies, 2013; Allen et al., 2017; Cortese, Amano, Koizumi, Kawato, \& Lau, 2016; Rouault et al., 2018) which suggests that this can be modulated in a domain-general manner.

The reported results of previous attempts to improve metacognitive ability through various combinations of training, systematic teaching and feedback in experimental settings with 
healthy participants have been equivocal (Bol, Hacker, O' Shea, \& Allen, 2005; Nietfeld and Schraw, 2002; Renner and Renner, 2001; Sharp, Cutler, \& Penrod, 1988). Similarly, a systematic review (Bhome, Berry, Huntley, \& Howard, 2018) found that cognitive training, mindfulness, group psychological, lifestyle and pharmacological interventions (Hoogenhout, de Groot, van der Elst, \& Jolles, 2012; Pereira-Morales, Cruz-Salinas, Aponte, \& PereiraManrique, 2017; Oh, Seo, Lee, Song, \& Shin, 2018; Scogin, Storandt, \& Lott, 1985; Valentijn et al., 2005; Smart, Segalowitz, Mulligan, Koudys, \& Gawryluk, 2016; Small et al., 2005; Brautigam et al., 1998; Zhu et al., 2016) did not significantly alter global and subjective measures of metacognitive ability in people with subjective cognitive decline (SCD). The SCD literature is relevant because more than half of all patients with SCD will meet criteria for FCD (Larner, 2018a), but extrapolating findings between the two remains difficult due to the presence of a significant minority of patients with SCD who have underlying pre-clinical neurodegenerative cognitive disorders (Hessen et al., 2017). There is only one randomised controlled study investigating treatments for FCD that used metacognition as a primary outcome. In this study, Metternich and colleagues (Metternich, Schmidtke, Harter, Dykierek, \& Hull, 2010) found that a group psychological intervention comprising psychoeducation and cognitive restructuring as well as stress reduction and relaxation techniques led to significant improvements in MSE scores at six month follow-up. Overall, due to a lack of evidence and consensus, combinations of the above interventions tend to be used somewhat arbitrarily in clinical practice to try to improve 'global' metacognition and reduce distress.

Recently, a novel and mechanistically plausible potential treatment option has appeared. Carpenter and colleagues (Carpenter et al., 2019) demonstrated that it is possible to systematically improve metacognitive ability using a computer-based training paradigm. Over eight training sessions spread over two weeks, healthy participants completed two perceptual discrimination tasks. After each trial, participants provided a confidence rating for their decisions. Task difficulty level was adjusted on an individual basis so that the rate of correct responses was uniform for all participants, thereby ensuring that first-order 
performance was not a confounding factor. Participants in the experimental group were given feedback on their metacognitive ability (i.e. how closely their confidence matched their perceptual performance) while those in the control group only received feedback on their actual performance (i.e. how often they were correct). The experimental group showed significant improvement in metacognitive ability compared to the control group. Interestingly, the poorer metacognitive ability was at baseline, the greater the benefits of training which suggests that the training may be even more effective in FCD patients who are likely to have greater metacognitive impairment. Further, improvement in metacognitive performance generalised to an untrained task in another domain (recognition memory). Generalisation of improvements in metacognition would be a useful effect of metacognitive training in FCD, where patients report cognitive difficulties in a range of cognitive domains.

\section{Future directions}

Metacognitive deficits have been suggested as a unifying mechanism in FCD. Here, we have tried to integrate this hypothesis with existing pathophysiological models of illness in other FNDs. The available evidence for metacognitive deficits in FCDs is limited. There is an urgent need to investigate whether people with FCD have impaired "local" metacognitive sensitivity when measured objectively in the laboratory using recently developed techniques (Fleming and Lau, 2014). If this is the case, further experimental work is required to see whether metacognitive ability can be trained in this patient group using mechanistically plausible translational treatments such as the computerised metacognitive training paradigm described above. As this is such a novel area, exploratory studies are required to establish the efficacy of metacognitive training in improving metacognitive ability in people with FCD and evaluating whether these improvements are sustained and lead to a reduction in FCD symptoms with an associated improvement in functioning. In the longer term, these findings would inform the design and conduct of clinical trials evaluating the clinical effectiveness of such interventions. Further, if functional imaging studies pre- and post-metacognitive training demonstrated plasticity in brain regions involved in metacognition, such as the fronto-parietal 
networks, this would provide further evidence for our understanding of the pathogenesis and treatment of FCD at the neural level. 


\section{Contributors}

$\mathrm{RH}, \mathrm{RB}$ and $\mathrm{JH}$ conceived this paper. $\mathrm{RB}$ drafted the paper with all authors $(\mathrm{RH}, \mathrm{AM}, \mathrm{SF}$, $\mathrm{JH}$ ) critically reviewing it and suggesting amendments to the content and structure prior to submission. All authors take responsibility for the integrity of the paper.

\section{Declarations of interest}

None of the authors declare any conflicts of interest.

\section{Funding}

This research received no specific grant from any funding body. RB is supported by a NIHR Academic Clinical Fellowship. JH and RH are supported by the NIHR UCLH BRC. AM is supported by the Mental Health and Justice Project funded by the Wellcome Trust (203376/2/16/Z). The Wellcome Centre for Human Neuroimaging is supported by core funding from the Wellcome Trust (203147/Z/ 16/Z). SMF is supported by a Sir Henry Dale Fellowship jointly funded by the Wellcome Trust and the Royal Society (206648/Z/17/Z). 


\section{References}

Allen, M., Glen, J. C., Mullensiefen, D., Schwarzkopf, D. S., Fardo, F., Frank, D., Rees, G. (2017). Metacognitive ability correlates with hippocampal and prefrontal microstructure. Neuroimage, 149, 415-423. doi:10.1016/j.neuroimage.2017.02.008

American Psychiatric Association. (2013) Diagnostic and Statistical Manual of Mental Disorders, Fifth Edition (DSM-5TM). Arlington, Virginia: American Psychiatric Press, Inc.

Bailey, C., Poole, N., \& Blackburn, D. J. (2018). Identifying patterns of communication in patients attending memory clinics: a systematic review of observations and signs with potential diagnostic utility. Br J Gen Pract, 68(667), e123-e138. doi: 10.3399/bjgp18x694601

Baird, B., Smallwood, J., Gorgolewski, K. J., \& Margulies, D. S. (2013). Medial and lateral networks in anterior prefrontal cortex support metacognitive ability for memory and perception. J Neurosci, 33(42), 16657-16665. doi:10.1523/JNEUROSCI.0786-13.2013

Bell, S., Harkness, K., Dickson, J. M., \& Blackburn, D. (2015). A diagnosis for pound55: what is the cost of government initiatives in dementia case finding. Age Ageing, 44(2), 344-345. doi:10.1093/ageing/afu205

Bharambe, V., \& Larner, A. J. (2018a). Functional cognitive disorders: demographic and clinical features contribute to a positive diagnosis. Neurodegenerative Disease Management, 8(6), 377-383. doi:10.2217/nmt-2018-0025

Bharambe, V., \& Larner, A. J. (2018b). Functional cognitive disorders: memory clinic study. Progress in Neurology and Psychiatry, 22(3), 19-22. doi:10.1002/pnp.509

Bhome, R., Berry, A. J., Huntley, J. D., \& Howard, R. J. (2018). Interventions for subjective cognitive decline: systematic review and meta-analysis. Bmj Open, 8(7). doi:ARTN e021610.1136/bmjopen-2018-021610 
Bhome, R., Huntley, J. D., Price, G., \& Howard, R. J. (2019) Clinical presentation and neuropsychological profiles of Functional Cognitive Disorder patients with and without comorbid depression. Cognitive Neuropsychiatry, 24(2), 152-164, doi:

$10.1080 / 13546805.2019 .1590190$

Bol, L., Hacker, D. J., O'Shea, P., \& Allen, D. (2005). The influence of overt practice, achievement level, and explanatory style on calibration accuracy and performance. Journal of Experimental Education, 73(4), 269-290. doi:Doi 10.3200/Jexe.73.4.269-290

Brautigam, M. R., Blommaert, F. A., Verleye, G., Castermans, J., Jansen Steur, E. N., \& Kleijnen, J. (1998). Treatment of age-related memory complaints with Ginkgo biloba extract: a randomized double blind placebo-controlled study. Phytomedicine, 5(6), 425-434. doi:10.1016/S0944-7113(98)80038-X

Carpenter, J., Sherman, M. T., Kievit, R. A., Seth, A. K., Lau, H., \& Fleming, S. M. (2019). Domain-general enhancements of metacognitive ability through adaptive training. J Exp Psychol Gen, 148(1), 51-64. doi:10.1037/xge0000505

Cortese, A., Amano, K., Koizumi, A., Kawato, M., \& Lau, H. (2016). Multivoxel neurofeedback selectively modulates confidence without changing perceptual performance. Nat Commun, 7, 13669. doi:10.1038/ncomms13669

Delis, D. C. \& Wetter, S. R. (2007). Cogniform Disorder and Cogniform Condition: proposed diagnoses for excessive cognitive symptoms. Arch Clin Neuropsychol, 22, 589-604. doi: 10.1016/j.acn.2007.04.001.

Dixon, R. A., Hultsch, D. F., \& Hertzog, C. (1988). The metamemory in adulthood (MIA) questionnaire. Psychopharmacology bulletin, 24(4), 671-688.

Donoso, M., Collins, A. G., \& Koechlin, E. (2014). Human cognition. Foundations of human reasoning in the prefrontal cortex. Science, 344(6191), 1481-1486.

doi:10.1126/science.1252254 
Edwards, M. J., Adams, R. A., Brown, H., Parees, I., \& Friston, K. J. (2012). A Bayesian account of 'hysteria'. Brain, 135(11), 3495-3512. doi: 10.1093/brain/aws129

Edwards, M. J., Fotopoulou, A., \& Pareés, I. (2013). Neurobiology of functional (psychogenic) movement disorders. Curr Opin Neurol, 26(4), 442-447. doi: $10.1097 /$ wco.0b013e3283633953

Elhadd, K., Bharambe, V. \& Larner, A.J. (2018). Functional cognitive disorders: can sleep disturbance contribute to a positive diagnosis? J. Sleep Disord. Ther, 7(3), 291. doi: $10.4172 / 2167-0277.1000291$

Fleming, S.M., \& Dolan, R.J. (2012). The neural basis of metacognitive ability. Philos Trans R Soc Lond B Biol Sci, 367(1594), 1338-49. doi: 1098/rstb.2011.0417

Fleming, S. M., Dolan, R. J., \& Frith, C. D. (2012). Metacognition: computation, biology and function. Philos Trans R Soc Lond B Biol Sci, 367(1594), 1280-1286.

doi:10.1098/rstb.2012.0021

Fleming, S. M., \& Lau, H. C. (2014). How to measure metacognition. Front Hum Neurosci, 8, 443. doi:10.3389/fnhum.2014.00443

Fleming, S. M., Weil, R. S., Nagy, Z., Dolan, R. J., \& Rees, G. (2010). Relating introspective accuracy to individual differences in brain structure. Science, 329(5998), 1541-1543. doi:10.1126/science.1191883

Hessen, E., Eckerstrom, M., Nordlund, A., Almdahl, I. S., Stalhammar, J., Bjerke, M., . . Wallin, A. (2017). Subjective Cognitive Impairment Is a Predominantly Benign Condition in Memory Clinic Patients Followed for 6 Years: The Gothenburg-Oslo MCI Study. Dementia and Geriatric Cognitive Disorders Extra, 7(1), 1-14. doi:10.1159/000454676 Hoogenhout, E. M., de Groot, R. H. M., van der Elst, W., \& Jolles, J. (2012). Effects of a comprehensive educational group intervention in older women with cognitive complaints: A 
randomized controlled trial. Aging \& Mental Health, 16(2), 135-144.

doi:10.1080/13607863.2011.598846

Larner A. J. (2018a). Dementia in Clinical Practice: A Neurological Perspective. Pragmatic studies in the Cognitive Function Clinic. London, UK: Springer.

Larner, A. J. (2018b). Dementia screening: a different proposal. Future Neurol, 13(4), 177179. doi:10.2217/fnl-2018-0018

Larner, A. J. (2018c). Metamemory: A construct with diagnostic utility in a cognitive disorders clinic? Int J Geriatr Psychiatry, 33(3), 553-554. doi:10.1002/gps.4766

McCurdy, L. Y., Maniscalco, B., Metcalfe, J., Liu, K. Y., de Lange, F. P., \& Lau, H. (2013). Anatomical coupling between distinct metacognitive systems for memory and visual perception. J Neurosci, 33(5), 1897-1906. doi:10.1523/JNEUROSCI.1890-12.2013

Metternich, B., Schmidtke, K., Harter, M., Dykierek, P., \& Hull, M. (2010). [Development and evaluation of a group therapy for functional memory and attention disorder]. Psychother Psychosom Med Psychol, 60(6), 202-210. doi:10.1055/s-0028-1103281

Metternich, B., Schmidtke, K., \& Hull, M. (2009). How are memory complaints in functional memory disorder related to measures of affect, metamemory and cognition? J Psychosom Res, 66(5), 435-444. doi:10.1016/j.jpsychores.2008.07.005

Nelson T.O., \& Narens, L. (1990). Metamemory: A theoretical framework and new findings. In G.H. Bower (Ed.), The Psychology of Learning and Motivation (pp. 125-173). San Diego, CA: Academic press

Nietfeld, J. L., \& Schraw, G. (2002). The effect of knowledge and strategy training on monitoring accuracy. Journal of Educational Research, 95(3), 131-142. doi:Doi $10.1080 / 00220670209596583$

Oh, S. J., Seo, S., Lee, J. H., Song, M. J., \& Shin, M. S. (2018). Effects of smartphonebased memory training for older adults with subjective memory complaints: a randomized 
controlled trial. Aging \& Mental Health, 22(4), 526-534.

doi:10.1080/13607863.2016.1274373

Paradise, M. B., Glozier, N. S., Naismith, S. L., Davenport, T. A., \& Hickie, I. B. (2011). Subjective memory complaints, vascular risk factors and psychological distress in the middle-aged: a cross-sectional study. BMC Psychiatry, 11(1), 108. doi: 10.1186/1471-244X11-108.

Pennington, C., Hayre, A., Newson, M., \& Coulthard, E. (2015). Functional Cognitive Disorder: A Common Cause of Subjective Cognitive Symptoms. J Alzheimers Dis, 48 Suppl 1, S19-24. doi:10.3233/JAD-150182

Pennington, C., Newson, M., Hayre, A., \& Coulthard, E. (2015). Functional cognitive disorder: what is it and what to do about it? Pract Neurol, 15(6), 436-444.

doi:10.1136/practneurol-2015-001127

Pereira-Morales, A. J., Cruz-Salinas, A. F., Aponte, J., \& Pereira-Manrique, F. (2018). Efficacy of a computer-based cognitive training program in older people with subjective memory complaints: a randomized study. International Journal of Neuroscience, 128(1), 1-9. doi:10.1080/00207454.2017.1308930

Rahnev, D., Koizumi, A., McCurdy, L. Y., D'Esposito, M., \& Lau, H. (2015). Confidence Leak in Perceptual Decision Making. Psychol Sci, 26(11), 1664-1680.

doi:10.1177/0956797615595037

Renner, C. H., \& Renner, M. J. (2001). But I thought I knew that: Using confidence estimation as a debiasing technique to improve classroom performance. Applied Cognitive Psychology, 15(1), 23-32. doi: 10.1002/1099-0720(200101/02)15:1<23::Aid-

Acp681>3.0.Co;2-J 
Reuber, M. \& Brown, R. J. (2017). Understanding psychogenic nonepileptic seizuresPhenomenology, semiology and the Integrative Cognitive Model. Seizure, 44, 199-205. doi: 10.1016/j.seizure.2016.10.029

Rouault, M., Seow, T., Gillan, C. M., \& Fleming, S. M. (2018). Psychiatric Symptom Dimensions Are Associated With Dissociable Shifts in Metacognition but Not Task Performance. Biol Psychiatry, 84(6), 443-451. doi:10.1016/j.biopsych.2017.12.017 Schmidtke, K. (1995). [Functional memory disorders. A study of 25 patients]. Nervenarzt, 66(5), 338-346.

Schmidtke, K. \& Metternich, B. (2009). Validation of two inventories for the diagnosis and monitoring of functional memory disorder. J Psychosom Res, 67, 245-51. doi: 10.1016/j.jpsychores.2009.04.005.

Schmidtke, K., Pohlmann, S., \& Metternich, B. (2008). The syndrome of functional memory disorder: definition, etiology, and natural course. Am J Geriatr Psychiatry, 16(12), 981-988. doi:10.1097/JGP.0b013e318187ddf9

Scogin, F., Storandt, M., \& Lott, L. (1985). Memory-skills training, memory complaints, and depression in older adults. J Gerontol, 40(5), 562-568.

Sharp, G. L., Cutler, B. L., \& Penrod, S. D. (1988). Performance Feedback Improves the Resolution of Confidence Judgments. Organizational Behavior and Human Decision Processes, 42(3), 271-283. doi:Doi 10.1016/0749-5978(88)90001-5

Small, G. W., Silverman, D. H. S., Siddarth, P., Ercoli, L. M., Miller, K. J., Lavretsky, H., . . Phelps, M. E. (2006). Effects of a 14-day healthy longevity lifestyle program on cognition and brain function. American Journal of Geriatric Psychiatry, 14(6), 538-545. doi:DOI 10.1097/01.JGP.0000219279.72210.ca

Smart, C. M., Segalowitz, S. J., Mulligan, B. P., Koudys, J., \& Gawryluk, J. R. (2016). Mindfulness Training for Older Adults with Subjective Cognitive Decline: Results from a Pilot 
Randomized Controlled Trial. Journal of Alzheimers Disease, 52(2), 757-774.

doi:10.3233/Jad-150992

Stone, J., Pal, S., Blackburn, D., Reuber, M., Thekkumpurath, P., \& Carson, A. (2015).

Functional (Psychogenic) Cognitive Disorders: A Perspective from the Neurology Clinic. J Alzheimers Dis, 48 Suppl 1, S5-S17. doi:10.3233/JAD-150430

Teodoro, T., Edwards, M. J., \& Isaacs, J. D. (2018). A unifying theory for cognitive abnormalities in functional neurological disorders, fibromyalgia and chronic fatigue syndrome: systematic review. J Neurol Neurosurg Psychiatry, 89(12), 1308-1319. doi:10.1136/jnnp-2017-317823

Valentijn, S. A. M., van Hooren, S. A. H., Bosma, H., Touw, D. M., Jolles, J., van Boxtel, M. P. J., \& Ponds, R. W. H. M. (2005). The effect of two types of memory training on subjective and objective memory performance in healthy individuals aged 55 years and older: a randomized controlled trial. Patient Education and Counseling, 57(1), 106-114. doi:10.1016/j.pec.2004.05.002

Wakefield, S. J., Blackburn, D. J., Harkness, K., Khan, A., Reuber, M., \& Venneri, A. (2018). Distinctive neuropsychological profiles differentiate patients with functional memory disorder from patients with amnestic-mild cognitive impairment. Acta Neuropsychiatr, 30(2), 90-96. doi:10.1017/neu.2017.21

World Health Organization. (2019, April). ICD-11 for Mortality and Morbidity Statistics. Retrieved from https://icd.who.int/browse11/l-m/en

Zhu, J., Shi, R., Chen, S., Dai, L., Shen, T., Feng, Y., . . Xing, G. (2016). The Relieving Effects of BrainPower Advanced, a Dietary Supplement, in Older Adults with Subjective Memory Complaints: A Randomized, Double-Blind, Placebo-Controlled Trial. EvidenceBased Complementary and Alternative Medicine, 2016, 16. doi:10.1155/2016/7898093 